\title{
Observation of a low-temperature acoustic anomaly in lanthanum lead zirconate titanate
}

\author{
Ghulam Shabbir, ${ }^{\text {a) }}$ Jae-Hyeon Ko, ${ }^{\text {b) }}$ and Seiji Kojima \\ Institute of Materials Science, University of Tsukuba, Tsukuba, Ibaraki-305-8573, Japan
}

(Received 29 September 2004; accepted 8 November 2004; published online 27 December 2004)

\begin{abstract}
High-resolution Brillouin scattering experiments have been performed to investigate the low temperature "aging" effect in lanthanum lead zirconate titanate (PLZT) relaxor ferroelectric ceramics. From the temperature dependence of the frequency shift of longitudinal acoustic mode, an acoustic anomaly was observed at $T_{0} \sim 46 \mathrm{~K}$ in these ceramics with clear "aging" effect below $T_{0}$. The results have been discussed in the light of stepwise freezing of the related degrees of freedom causing transition from proper to improper dipole glassy states at $T_{0}$. (C) 2005 American Institute of Physics. [DOI: 10.1063/1.1847710]
\end{abstract}

Relaxor ferroelectric materials have been under extensive investigations since their discovery, mainly because of their excellent dielectric, piezoelectric, pyroelectric, and electro-optic applications. ${ }^{1}$ These materials have substantial disorder in the valance and/or size of the cation sublattices. They display a diffuse phase transition, a strong frequency dispersion of the dielectric susceptibility, and an absence of macroscopic polarization at zero electric field. Relaxors resemble glassy materials, since they exhibit phenomena characterizing glasses, e.g., aging, memory, and rejuvenation., ${ }^{2,3}$ In a typical aging experiment, the slow reduction in the dynamic susceptibility is observed during a stay (a waiting time $t_{w}$ ) at a constant temperature $T_{A}$, below the glass transition temperature $T_{g}$. Although $T_{g}$ cannot be defined exactly in relaxors, they seem to be interesting materials because of their significant "aging" effect not only within the frozen regime, ${ }^{4}$ but also above the susceptibility maximum $T_{m}(\sim 350 \mathrm{~K}) .^{5}$

In spite of intensive studies, the mechanism, which leads to a low-temperature disordered frozen state in relaxor ferroelectrics, is not yet completely understood. Many recent investigations support the existence of qualitative equivalence between the nonergodic relaxor (NER) state appearing below a certain freezing temperature $T_{f}$ and the dipolar glass state. As to the concept of random fields, the observed dynamics in the NER phase (at $T<T_{f}$ ) of lead magnesium niobate (PMN) ceramics has been shown to result in glassy states. ${ }^{6}$ MicroRaman scattering studies of $\left(\mathrm{Pb}_{1-x} \mathrm{La}_{x}\right)\left(\mathrm{Zr}_{y} \mathrm{Ti}_{1-y}\right) \mathrm{O}_{3}$ PLZT $x /(y(1-y)$ ceramics also showed an agreement with the glassy character of the low temperature relaxor state. ${ }^{7}$ On the basis of freezing of superparelectric clusters, it was also proposed that relaxors could be considered as a dipolar glass. ${ }^{8,9}$ The low-temperature anomalies observed near $T \sim 45 \mathrm{~K}$ in PLZT-9/65/35 ceramic $^{10}$ were attributed to a dipole glass state. Therefore, it seems important to study the low temperature "aging" in relaxors to improve our present physical understanding of the glassy nature of these materials. Here we report the observation of a low temperature acoustic anomaly with significant "aging" effect in PLZT relaxor

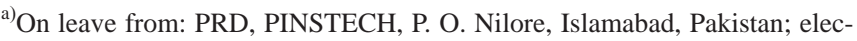
tronic mail: shabbir@ims.tsukuba.ac.jp

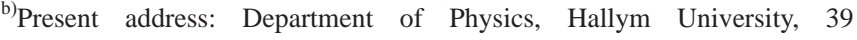
Hallymdaehak-gil, Chuncheon-Si, Gangwon-Do, 200-702, Korea.
}

ferroelectric ceramics measured by the high-resolution Brillouin scattering technique.

The material under study here is PLZT ceramic with two slightly different compositions, $x=0.10$ and $y=0.65$ (PLZT$10 / 65 / 35$ ), and $x=0.076$ and $y=0.72$ (PLZT-7.6/72/28). The parent PZT-65/35 is a normal ferroelectric below $T_{c}$ $\sim 627 \mathrm{~K}$. The partial substitution of $\mathrm{Pb}$ with $\mathrm{La}(7<x$ $<12)$ results in the formation of PMRs below $T_{B} \sim T_{c}$ ( $\sim 627 \mathrm{~K}$ for PZT-65/35), with a typical relaxor behavior.

The transparent ceramic samples were prepared by hotpressing in an oxygen atmosphere. Specimens were carefully polished to optical quality and loaded into the specimen holder for measurements. A $3+3$ pass Sandercock tandem Fabry-Perot interferometer was used to measure the Brillouin spectra by employing right angle (90-A) scattering geometry. The specimens were excited by an $\mathrm{Ar}^{+}$-ion laser with a wavelength of $514.5 \mathrm{~nm}$. A closed cycle helium refrigerator (RMC LTS-22) system was used for low temperature measurements. For each specimen two data sets were taken. First the Brillouin spectra were recorded by cooling the sample from 300 to $15 \mathrm{~K}$ and then heating to $\sim 80 \mathrm{~K}$ with no waiting

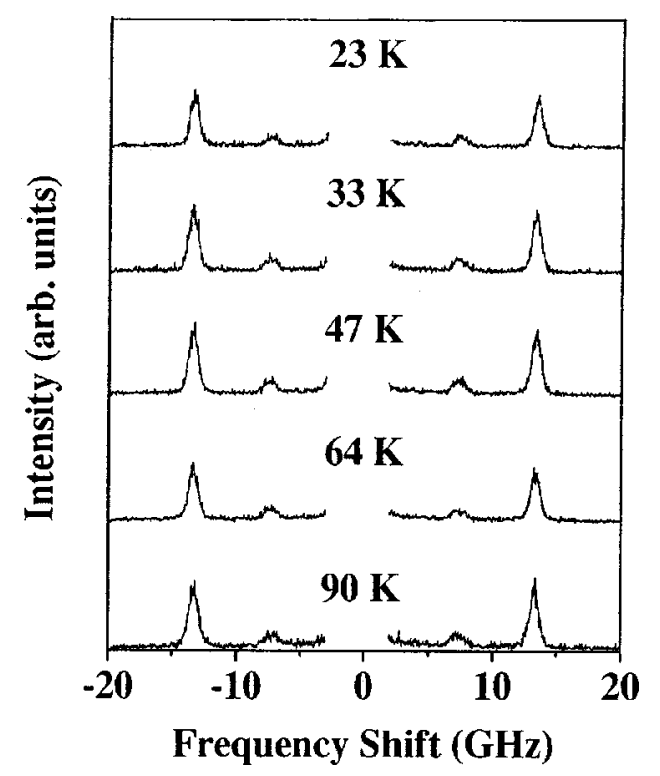

FIG. 1. Brillouin spectra of PLZT-10/65/35 ceramic at some selected temperatures measured in cooling run. 


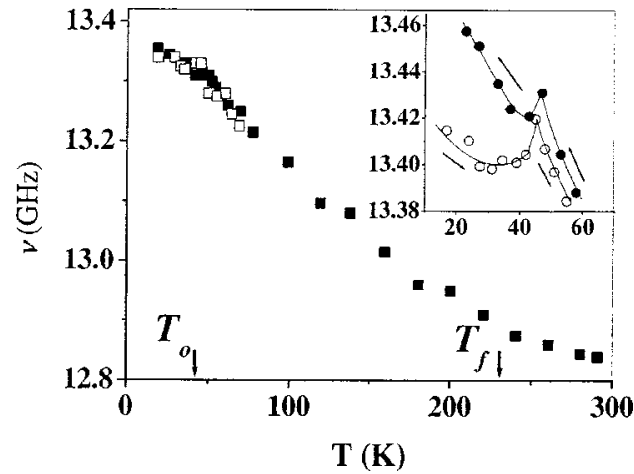

FIG. 2. Brillouin frequency shift $(\nu)$ of the LA mode of PLZT-10/65/35 ceramic as a function of temperature for both cooling (closed squares) and subsequent heating (open squares) runs $\left(t_{w}=0\right)$. The inset shows cooling and subsequent heating runs $\left(t_{w}=12 \mathrm{~h}\right.$ at $\left.15 \mathrm{~K}\right)$.

time $\left(t_{w}=0\right)$ at the lowest temperature. Second, the ceramics were cooled from room temperature to $15 \mathrm{~K}$ and the spectra were measured in heating run after waiting $\left(t_{w}=12 \mathrm{~h}\right)$ at 15 $\mathrm{K}$. All the experiments were done in zero external field with a temperature stability of $\pm 1 \mathrm{~K}$.

Typical Brillouin spectra associated with the acoustic phonon modes in PLZT-10/65/35 at some selected temperatures are plotted in Fig. 1. The Brillouin spectra of PLZT-7.6/ $72 / 28$ were similar and hence are not shown. Before each aging experiment the sample was brought to the reference temperature by cooling from room temperature at $1 \mathrm{~K} / \mathrm{min}$. These spectra consist of one longitudinal acoustic (LA) and one transverse acoustic (TA) mode. The instrumental function was assumed as a Gaussian and phonon peaks were fitted with Lorentzian function to obtain frequency shift $(\nu)$ and phonon damping. The resulting temperature dependent LA phonon mode frequency shift for the two ceramics is plotted in Figs. 2 and 3, respectively.

The Brillouin frequency shifts of the LA and TA modes denoted by $\nu_{\mathrm{LA}}$ and $\nu_{\mathrm{TA}}$, respectively, are related to the elastic constants $c_{i j}$ through the corresponding sound velocities $V_{\mathrm{LA}}$ and $V_{\mathrm{TA}}$ by

$$
\frac{2 \pi \nu_{\mathrm{LA}}}{q}=V_{\mathrm{LA}}=\left[\frac{c_{11}}{\rho}\right]^{1 / 2},
$$

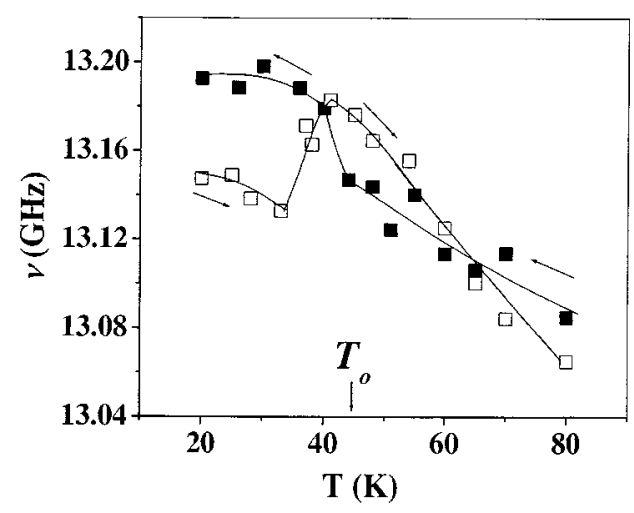

FIG. 3. Temperature dependence of the Brillouin frequency shift $(\nu)$ of the LA mode of PLZT-7.6/72/28 ceramic in cooling (closed squares) and subsequent heating (open squares) runs $\left(t_{w}=12 \mathrm{~h}\right.$ at $15 \mathrm{~K}$ ), where the solid curves are guides for the eye.

Downloaded 30 Nov 2009 to 130.158 .56 .123 . Redistribution subje

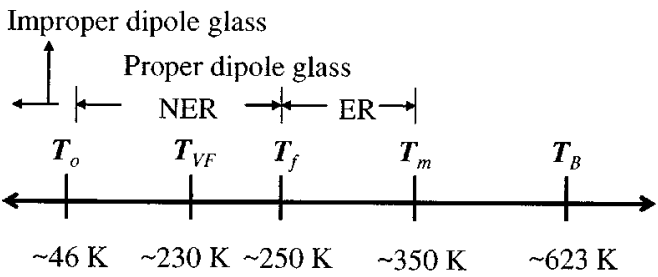

FIG. 4. Sequence of well-known physical anomalies in PLZT relaxor ferroelectrics.

$$
\frac{2 \pi \nu_{\mathrm{TA}}}{q}=V_{\mathrm{TA}}=\left[\frac{c_{44}}{\rho}\right]^{1 / 2},
$$

where $\rho$ is the sample density, $7863 \mathrm{Kg} / \mathrm{m}^{3}$, and $q$ is the scattering wave vector.

Figure 2 shows temperature dependence of the LA mode frequency shift of PLZT-10/65/35. It can be seen that the Brillouin frequency shift increases with decreasing temperature with a change in slope at $T_{0} \sim 46 \mathrm{~K}$. Data follow the same path (Fig. 2) during heating back from the lowest temperature $(15 \mathrm{~K})$ without waiting time $\left(t_{w}=0\right)$. The results were similar for the other ceramic and hence are not shown. Figure 3 and the inset of Fig. 2 clearly exhibit the "aging" effect when the data were measured during heating after a waiting time of $t_{w}=12 \mathrm{~h}$ at $15 \mathrm{~K}$. This anomaly is relatively small in magnitude but the results were reproducible. TA mode frequency shift also showed similar increasing trend with lowering temperature but the anomaly at $T_{0} \sim 46 \mathrm{~K}$ could not be observed clearly.

In PLZT relaxor, the dynamics of various physical anomalies (Fig. 4) is governed by the appearance of PMRs at Burn's temperature. PMRs grow in size with decreasing temperature and ergodic relaxor (ER) state appears below $T_{m}$. Further lowering in temperature is accompanied by the freezing of randomly oriented PMRs at $T_{f}$ and onset of NER state as evidenced by splitting between the field-cooled and the zero-field cooled susceptibility in both PMN and PLZT-8/ $65 / 35$ relaxor ceramics. ${ }^{11}$ This breaking of ergodicity is similar to the one observed in other glassy systems including dipolar and quadrupolar glasses. ${ }^{12}$ But still at $T \leqslant T_{f}$ these random polarizations can be put in order by applying sufficient external field $\left(E \geqslant E_{C} \sim 5 \mathrm{kV} / \mathrm{cm}\right)$ causing relaxor to ferroelectric phase transition. The polarization fluctuations freeze completely at $T_{\mathrm{VF}}$ where the maximum relaxation time $\tau_{\max } \sim \omega^{-1}$ diverges according to the Vögel-Fulcher relation $\tau_{\max }=\tau_{0} \exp \left[E_{a} / k_{B}\left(T-T_{f}\right)\right]^{13}$ and random polarizations associated with PMRs cannot be put into ordered state even by applying external field for $T \leqslant T_{\mathrm{VF}}$. At $T_{0}$, there are some remaining degrees of freedom that freeze as discussed in the following.

In traditional spin glasses, aging effect is found below the glass transition temperature $T_{g}$, and this effect is considered a signature of the spin glass state. In PLZT the definition of glass transition is not clear, but, by analogy with other glassy systems, it should be very close to and in any case below the temperature at which relevant degrees of freedom appear to freeze. Observation of memory and multiple aging stages in PLZT relaxor ${ }^{5}$ above $T_{f}$ in contrast to other glassy systems reflects the complexity of the situation. Moreover, aging has been reported to occur even above $T_{m}$ in PLZT, and more complex phenomena than in other relaxor ferro- 
electric, e.g., PMN-PT, has been assigned to these multiple aging mechanisms. ${ }^{4}$

A boson peak in the Raman spectra, anomalies in the dielectric permittivity and in the pyroelectric current at $T$ $\sim 45 \mathrm{~K}$ were reported in PLZT-9/65/35. ${ }^{10}$ The appearance of boson peak indicates the excess part of vibrational density of states, which is closely related to the existence of medium range order. ${ }^{14}$ The freezing of remaining degrees of freedom induces the medium range order, and it causes the acoustic anomaly. With this background the acoustic anomaly at $T_{0}$ might be anticipated by that freezing process in PLZT relaxor takes place in steps. First, PMRs freeze partially at $T_{f}$ and then completely at $T_{\mathrm{VF}}$ but the degrees of freedom related to A-site dynamical disorder prevail. At $T_{0}$ these remaining degrees of freedom also freeze and a transition to an "improper dipole glass" state occurs. It is proposed that the low temperature freezing process gives rise to a shift of the charge center because of relative displacements of cations and anions, and hence a change in polarization which causes an acoustic anomaly at $T_{0}$.

High-resolution Brillouin scattering investigations were performed on PLZT relaxor ferroelectric ceramics in the temperature range from 300 to $15 \mathrm{~K}$. A low temperature acoustic anomaly was observed at $T_{0} \sim 46 \mathrm{~K}$ with clear aging effect below $T_{0}$. This might be associated with the freezing of degrees of freedom at $T_{0}$ related to A-site dynamical disorder with transition from proper to improper dipole glassy state.
G.S. is thankful to the Japanese Government for a MONBU-KAGAKUSHO scholarship. The authors are also grateful to the 21st Century COE program under the Japanese Ministry of Education, Culture, Sports, Science and Technology, Japan for providing financial support for this work.

${ }^{1}$ G. H. Haertling, Ferroelectrics 75, 25 (1987).

${ }^{2}$ E. V. Colla, L. K. Chao, M. B. Weissman, and D. Viehland, Phys. Rev. Lett. 85, 3033 (2000).

${ }^{3}$ O. Kircher and R. Böhmer, Eur. Phys. J. B 26, 329 (2002).

${ }^{4}$ E. V. Colla, L. K. Chao, and M. B. Weissman, Phys. Rev. B 63, 134107 (2001), and references therein.

${ }^{5}$ F. Cordero, F. Craciun, A. Franco, D. Piazza, and C. Galassi, Phys. Rev. Lett. 93, 097601 (2004).

${ }^{6}$ M. D. Glinchuk and R. Farhi, J. Phys.: Condens. Matter 8, 6985 (1996).

${ }^{7}$ M. El Marssi, R. Farhi, J.-L. Dellis, M. D. Glinchuk, L. Seguin, and D. Viehland, J. Appl. Phys. 83, 537 (1998).

${ }^{8}$ L. E. Cross, Ferroelectrics 76, 241 (1987).

${ }^{9}$ D. Viehland, S. J. Jang, L. E. Cross, and M. Wuttig, Phys. Rev. B 46, 8003 (1992).

${ }^{10}$ R. Farhi, M. El Marssi, J.-L. Dellis, M. D. Glinchuk, and V. A. Stephanovich, Europhys. Lett. 46, 351 (1999).

${ }^{11}$ Z. Kutnjak, C. Filipic, R. Pric, A. Levstik, R. Farhi, and M. El Marssi, Phys. Rev. B 59, 294 (1999).

${ }^{12}$ A. Levstik, C. Filipic, Z. Kutnjak, I. Levstik, R. Pirc, B. Tadic, and R. Blinc, Phys. Rev. Lett. 66, 2368 (1991).

${ }^{13}$ G. Shabbir, J.-H. Ko, S. Kojima, and Q.-R. Yin, Appl. Phys. Lett. 82, 4696 (2003).

${ }^{14}$ E. Duval, A. Boukenter, and B. Champagnon, Phys. Rev. Lett. 56, 2052 (1986). 